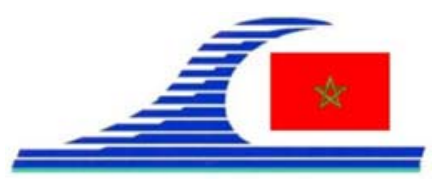

Conférence Méditerranéenne Côtière et Maritime EDITION 2, TANGER, MAROC (2011)

Coastal and Maritime Mediterranean Conference

Disponible en ligne - http://www.paralia.fr-Available online

\title{
Oscillations induites dans un port à parois totalement réfléchissantes sollicité par une houle incidente régulière ou irrégulière
}

\author{
Noureddine DABSSI ${ }^{1,2}$, Mohamed CHAGDALI ${ }^{1}$
}

1. LCSM, Faculté des Sciences Ben M’Sik, Casablanca, Maroc.

2. Marine Royale, Casablanca, Maroc.

m_chagdali@hotmail.com

\section{Résumé :}

On présente une étude numérique, basée sur la Méthode des Eléments Frontières (BEM), des oscillations induites dans un port sollicité par une houle régulière ou irrégulière. Le problème est formulé par des équations intégrales et les résultats sont présentés en termes de coefficient d'amplification (rapport de l'amplitude d'agitation et de l'amplitude de la houle incidente). La procédure est validée par une simulation concernant le cas test d'un port composé de deux bassins circulaires. Par la suite, cette procédure a été appliquée sur plusieurs configurations des ports et en particulier sur le Port de Tanger Méditerranéen.

\section{Mots-clés :}

Agitation portuaire -Résonance portuaire - Fonction de Green

\section{Introduction}

Les structures marines ou navales dont la surface libre ou une portion de cette surface est confinée entre des frontières fermées ou ouvertes, sollicités par une houle incidente, présentent des réponses particulières faisant apparaître des oscillations ayant des périodes propres notamment celles correspondantes aux modes de Helmholtz. Ce phénomène connu sous le nom de résonance portuaire, seiche ou déferlante portuaire, peut causer une navigation aléatoire et des dommages assez signifiants pour les navires amarrés et les structures adjacentes, notamment lorsque les périodes propres de résonance portuaire avoisinent les périodes propres du système navire-amarre. Ce problème a fait l'objet de plusieurs recherches (LEE, 1971; HWANG \& TUCK, 1970 ; ISAACSON \& QU, 1990 ; DABSI, 2008). Dans ce travail, on va étudier ce problème d'une manière numérique avec des applications sur quelques ports du Maroc.

\section{Equation de mouvement}

Le problème de l'agitation portuaire peut être formulé dans le cadre de la théorie potentielle de la houle. Il s’agit de déterminer le potentiel de vitesses $\Phi$, tel que :

$$
\Phi(x, y, z ; t)=\mathfrak{R}\left(\phi(x, y, z) e^{-i \omega t}\right) \quad \text { avec }: \phi(x, y, z)=\frac{i}{\omega} \varphi(x, y) Z(z)
$$


La connaissance de la Mer :

un vecteur du développement durable en Méditerranée

Où $\omega$ est la fréquence angulaire $(\omega=2 \pi / T)$, avec $T$ la période de la houle, $\varphi(x, y)$ est la fonction d'onde décrivant la variation du potentiel dans le plan $x$ et $y$.

A partir de l'équation de continuité, on peut montrer que le potentiel bidimensionnel $\varphi$ vérifie le système d'équations suivant (DABSI, 2008) :

$\left\{\begin{array}{l}\frac{d^{2} Z}{d z^{2}}-k^{2} Z=0 \\ \frac{\partial^{2} \varphi}{\partial x^{2}}+\frac{\partial^{2} \varphi}{\partial y^{2}}+k^{2} \varphi=0\end{array} \quad \omega^{2}=g k \tanh (k h)\right.$

La condition d'imperméabilité au de transmissions sur les parois (ISAACSON \& QU, 1990) s'écrit :

$\frac{\partial \phi_{2}}{\partial n}+\gamma k \phi_{2}=0$ sur $\Gamma$, avec : $\gamma=i \frac{1-K_{r}}{1+K_{r}}$

Où $n$ est la normale au contour $\Gamma, \gamma$ est le coefficient de transmission, $K_{r}$ est le coefficient de réflexion des parois.

\section{Procédure de résolution}

La procédure de résolution est constituée des étapes suivantes :

- Le domaine d'étude est subdivisé en deux régions : une région de l'océan infini (région I) et une région délimitée par les frontières du port (région II).

- La ligne de côte qui fait partie de la région I est constitué de paroi totalement réfléchissante.

- Le potentiel $\varphi_{1}$ est formulé dans la région I en fonction des dérivées normales inconnues $\partial \varphi_{1} / \partial n$ à l'entrée du port. De la même façon le potentiel $\varphi_{2}$ est exprimé dans la région II en fonction des dérivées normales inconnues $\partial \varphi_{2} / \partial n$ à l'entrée du port. On exprime donc la continuité de la pression et de la vitesse; $\varphi_{1}=\varphi_{2}$ et $\partial \varphi_{1} / \partial n=\partial \varphi_{2} / \partial n$.

- Une fois que la dérivée normale $\partial \varphi_{2} / \partial n$ est connue, le potentiel $\varphi_{2}$ peut être déterminé dans la région II à l'intérieur du port.

\subsection{Formulation numérique}

En appliquant la seconde identité de Green, le problème précédent peut être écrit sous forme intégrale de la manière suivante :

$b \varphi_{j}(P)=\int_{\Omega_{j}}\left[\varphi_{j}(Q) \frac{\partial G(P, Q)}{\partial n_{Q}}-G(P, Q) \frac{\partial \varphi_{j}(Q)}{\partial n_{Q}}\right] d s$

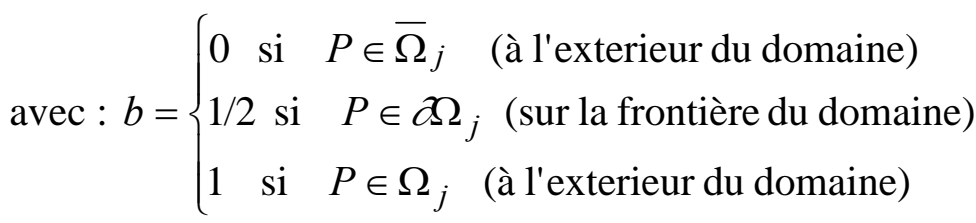


$n_{Q}$ étant la normale à la frontière et $d s$ est la longueur d'un segment de ligne sur la frontière. La fonction de Green étant la fonction de Hankel de $1^{\text {ère }}$ espèce : $H_{0}{ }^{(1)}(k r)$.

$r_{p q}=\sqrt{\left(x_{p}-x_{q}\right)^{2}+\left(y_{p}-y_{q}\right)^{2}}$

\subsection{Réponse du port sollicité par une houle régulière}

Le coefficient d'amplification $R$ est défini par le rapport de l'amplitude de l'élévation de la surface libre $\zeta_{2}(x, y ; t)$ en un point quelconque du port et la somme des amplitudes des houles incidentes et réfléchies sur la côte extérieure du port (entrée du port fermée).

$$
R=\frac{\left|\zeta_{2}(x, y ; t)\right|}{\left|A_{i}\left(\varphi_{i}(x, y)+\varphi_{r}(x, y)\right) e^{-i \omega t}\right|}
$$

\subsection{Réponse du port sollicité par une houle irrégulière}

La houle aléatoire qui peut être représentée par un spectre de houle incidente $S_{I}(\omega)$. Le spectre utilisé est celui de Pierson-Moskowitz est qui est défini par :

$$
S_{I}(\omega)=\frac{\alpha g^{2}}{\omega^{5}} \exp \left[-\beta\left(\frac{g}{V \omega}\right)^{4}\right]
$$

Le spectre des dénivellations en un point quelconque du port est :

$S_{R}(\omega)=|R(\omega)|^{2} S_{I}(\omega)$

La hauteur significative $H_{m 0}=4,004 m_{0}^{1 / 2}$ de l'agitation dans le port est directement déterminée à partir du moment d’ordre zéro $m_{0}$, tel que :

$$
m_{n}=\int_{0}^{\infty} \omega^{n} S_{R}(\omega) d \omega
$$

\section{Résultats}

En absence des données sur la nature des parois des ports simulés dans ce travail, nous avons considéré que les parois sont totalement réfléchissantes. Cette situation correspond au cas extrême de l'agitation.

\subsection{Port composé de deux bassins circulaires}

Un calcul a été effectué pour un port composé de deux bassins circulaires connectés par une ouverture de $10^{\circ}$ et dont l'entrée face à la mer est de $60^{\circ}$. Ce cas a été étudié expérimentalement par LEE (1971) Sur la figure 1 on présente les courbes de réponse numériques et expérimentales. En ordonnée le coefficient d'amplification et en abscisse le paramètre nombre d'onde $k a$ (produit du nombre d'onde par le rayon du port circulaire). On constate une bonne concordance entre les résultats théoriques et expérimentaux. 
La connaissance de la Mer :

un vecteur du développement durable en Méditerranée

\subsection{Port de Casablanca}

Les caractéristiques réelles du contour ainsi que les principaux secteurs de ce port sont exposées dans la référence DABSI (2008). Ici on considère la situation extrême de l'agitation par le fait que les toutes les parois sont totalement réfléchissantes. Sur la figure 2, on présente les résultats pour le port de Casablanca (Coefficient d'amplification en fonction du nombre d'onde $\mathrm{k}\left(\mathrm{m}^{-1}\right)$ ). Sur la figure 3 , on présente les résultats de la superposition de l'agitation avec le spectre de la houle irrégulière pour un vent de 25 nœuds.

\subsection{Port de Tanger Med}

Les principaux secteurs de ce port sont exposés dans la référence DABSI (2008). Les parois du contour du port sont considérées être totalement réfléchissantes. Sur la figure 4, on présente l'agitation dans le port de Tanger-méditerranée en plusieurs points (coefficient d'amplification en fonction du paramètre nombre d'onde $k a$, qui est le produit du nombre d'onde par l'ouverture du port).
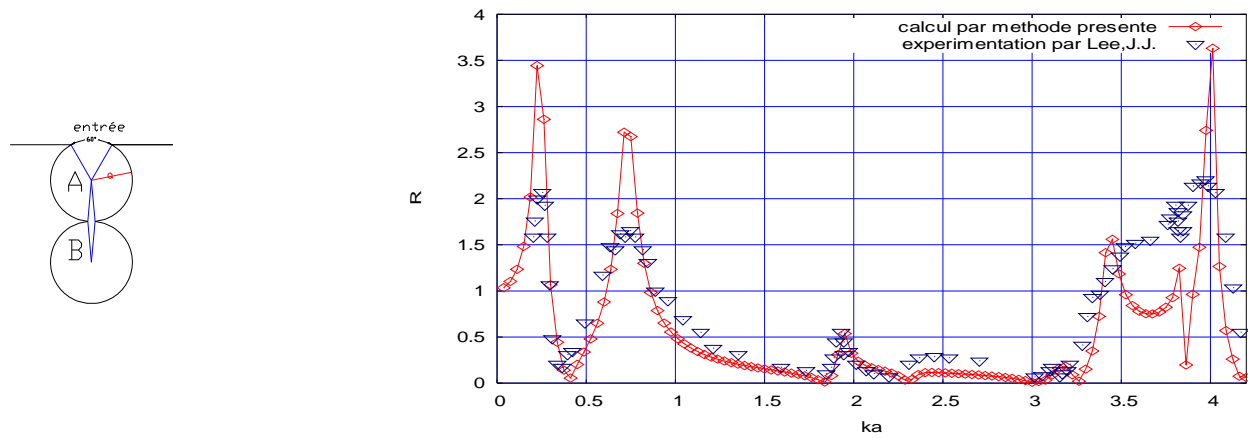

Figure 1. Agitation dans un port bi-circulaire au centre du cercle A.
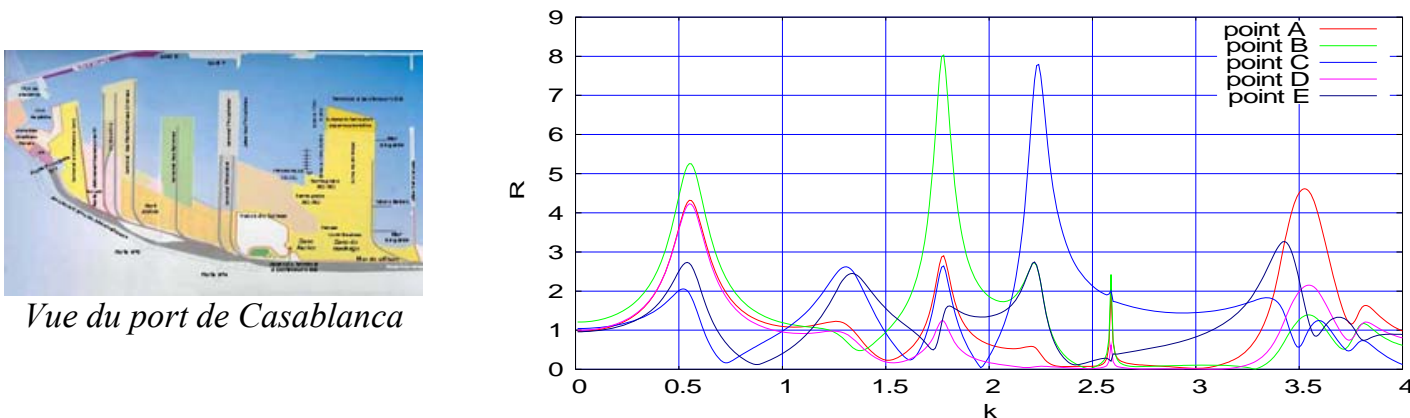

Figure 2. Coefficient d'amplification dans plusieurs points du port de Casablanca. Les points $A, B, C, D$ et E sont situés en différentes positions de la ligne perpendiculaire à l'entrée. 


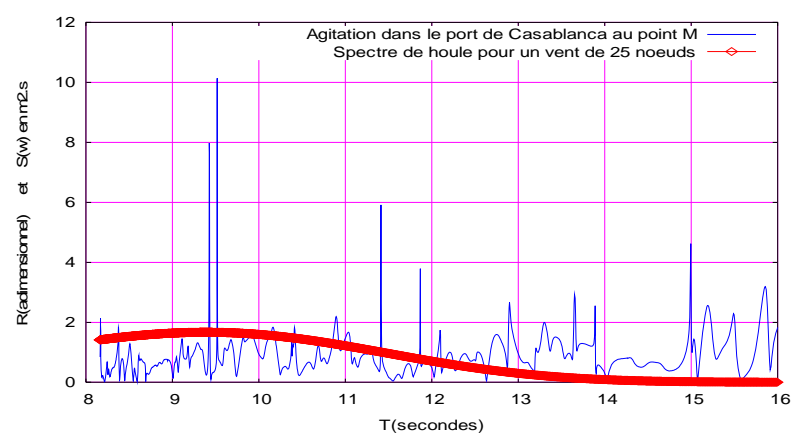

Figure 3. Spectre de la houle irrégulière correspondant à un vent de 25 nœuds.

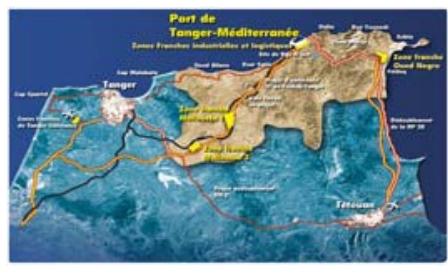

Vue du port de

Tanger-méditerranée

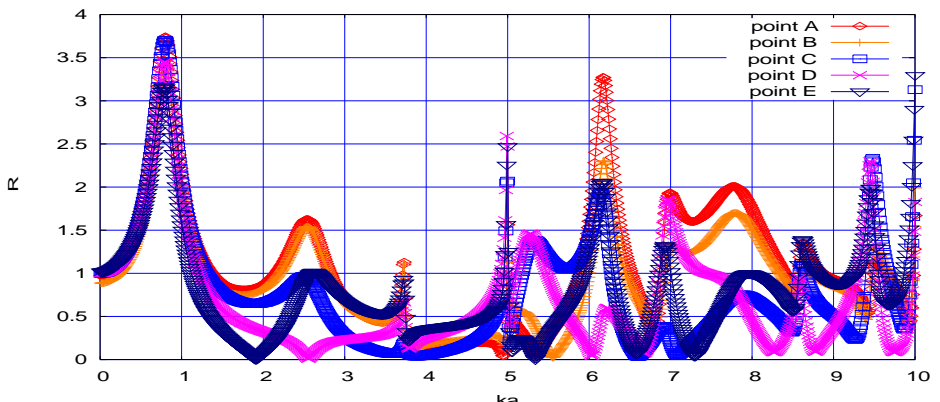

Figure 4. Agitation dans le port de Tanger-méditerranée.

Les points $A, B, C, D$ et $E$ sont situés en différentes positions de la ligne perpendiculaire à l'entrée.

\section{Conclusion}

Le modèle mathématique qui a été présenté a été développé en utilisant la méthode des éléments frontières pour la détermination de l'élévation de la surface libre dans un port de géométrie quelconque sollicité par une houle régulière ou irrégulière. En absence des données sur la nature des contours des ports simulés, on a considéré que les parois sont totalement réfléchissantes. Les résultats de ce modèle numérique comparés au cas test d'un port composé de deux bassins circulaires montrent la bonne concordance. Des applications de ce code de calcul ont été effectuées sur des ports au Maroc. Ces applications surestiment l'agitation à cause de l'hypothèse des parois totalement réfléchissantes.

\section{References bibliographiques}

DABSI N. (2008). Contribution à l'étude des modes de résonance des structures fixes ou flottantes en hydrodynamique marine et navale. Thèse de Doctorat soutenue le 20 décembre 2008 à la Faculté des Sciences Ben M’Sik Casablanca, Maroc.

HWANG L.S., TUCK E.O. (1970). On the oscillations of harbours of arbitrary shape. Journal of Fluid Mechanics, Vol. 42, pp 447-464. doi:10.1017/S0022112070001404 
La connaissance de la Mer :

un vecteur du développement durable en Méditerranée

ISAACSON M., QU S. (1990). Waves in harbour with reflecting boundaries. Coastal Engineering, Vol. 14, pp 193-214. doi:10.1016/0378-3839(90)90024-Q

LEE J.J. (1971). Wave induced oscillations in harbours of arbitrary geometry. Journal of Fluid Mechanics, Vol. 45, part 2, pp 375-394. 